\title{
Relations among the lowest degree of the Jones polynomial and geometric invariants for a closed positive braid
}

Tomomi Kawamura

\begin{abstract}
By means of a result due to Fiedler, we obtain a relation between the lowest degree of the Jones polynomial and the unknotting number for any link which has a closed positive braid diagram. Furthermore, we obtain relations between the lowest degree and the slice euler characteristic or the four-dimensional clasp number.
\end{abstract}

Mathematics Subject Classification (2000). 57M25.

Keywords. Unknotting number, four-dimensional clasp number, slice Euler characteristic, Jones polynomial.

\section{Introduction and a main result}

A link is a closed oriented 1-manifold smoothly embedded in the 3 -sphere $S^{3}$ and a knot is a link with one component. Let $L$ be a link with $r$ components. The Jones polynomial $V(L ; t) \in \mathbb{Z}\left[t^{\frac{1}{2}}, t^{-\frac{1}{2}}\right]$ is an ambient isotopy invariant of oriented links $L$ and is defined by the following relations [5]:

$$
\begin{aligned}
& V(\text { a trivial knot; } t)=1
\end{aligned}
$$

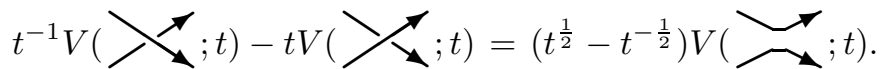

We denote by min $\operatorname{deg} V(L ; t)$ the lowest degree of $V(L ; t)$. In [4], Fiedler determined the lowest degree of the Jones polynomial for some links. In this paper, we review his result in Section 3, and obtain some relations among the lowest degree of the Jones polynomial and the following invariants for a closed positive braid by his result.

The unknotting number $u(L)$ of the link $L$ is the minimal number of crossing changes needed to create the trivial link with $r$ components. Let $L$ be a link and $F \subset D^{4}$ a smooth, oriented 2-manifold with $\partial F=L$, where $D^{4}$ is the 4ball bounded by $S^{3}$. We suppose that $F$ has no closed components, but $F$ is not assumed to be connected. The slice euler characteristic $\chi_{s}(L)$ of the link $L$ is

\footnotetext{
The author is supported by JSPS Research Fellowships for Young Scientists.
} 
the greatest value of the euler characteristic $\chi(F)$ for such 2-manifolds $F \subset D^{4}$. We denote by $c_{s}(L)$ the minimum number of the double points for transversely immersed disks in $D^{4}$ with boundary $L$ and with only finite double points as singularities, and we shall call this invariant the 4-dimensional clasp number. In [8], the author showed that the following inequality holds for any oriented link $L$ with $r$ components:

$$
u(L) \geq c_{s}(L) \geq \frac{1}{2}\left(r-\chi_{s}(L)\right)
$$

A closed positive braid diagram is a closed braid diagram with no negative crossings. A closed positive braid or a closed positive braid link is an oriented link which has a closed positive braid diagram. Let $D$ be a link diagram. Let $s(D)$ be the number of the Seifert circles and $x(D)$ be the number of the crossings of $D$. Let $L$ be an $r$-component link with a closed positive braid diagram $D_{L}$. Boileau and Weber [2], and Rudolph [14] showed that the inequality $u(L) \leq \frac{1}{2}\left(r-s\left(D_{L}\right)+\right.$ $\left.x\left(D_{L}\right)\right)$ holds for such a link $L$. It was conjectured that the equality

$$
u(L)=\frac{1}{2}\left(r-s\left(D_{L}\right)+x\left(D_{L}\right)\right)
$$

holds for an $r$-component link $L$ with a closed positive braid diagram $D_{L}$. (cf. $[2],[11],[14])$. In [8], the author proved that an inequality stronger than that conjectured by Bennequin [1] holds for any link diagram, and showed that the equality (2) and the equality $u=c_{s}=\frac{1}{2}\left(r-\chi_{s}\right)$ hold for any closed positive braid with $r$ components. We review these results in Section 2 .

By combining a result of Fiedler, that is Theorem 3.1, with a result including the equality (2), that is Theorem 2.1, we have the following result.

Theorem 1.1. Let $L$ be a closed positive braid link with $r$ components. Then we have

$$
\min \operatorname{deg} V(L ; t)=u(L)-\frac{1}{2}(r-1) .
$$

Furthermore we have min $\operatorname{deg} V(L ; t)=c_{s}(L)-\frac{1}{2}(r-1)$ and $\min \operatorname{deg} V(L ; t)=$ $\frac{1}{2}\left(1-\chi_{s}(L)\right)$ for such a link $L$.

In section 2, we survey the stronger Bennequin unknotting inequality and the equality (2) before the proof of Theorem 1.1. In Section 3, we review a result of Fiedler [4] and prove Theorem 1.1. In Section 4, we consider some relation between the lowest degree of the Jones polynomial and the slice euler characteristic for quasipositive links.

The author would like to thank Professor Toshitake Kohno for his encouragement and useful advice. She would like to thank Professor Hanspeter Kraft and the referee for their useful comments. 


\section{The Bennequin unknotting inequality}

In this section, we review results in [8], which we use in the proof of Theorem 1.1.

Let $D$ be an oriented link diagram. The writhe $w(D)$ is the value defined by the number of positive crossings minus the number of negative crossings of $D$. We give signs to Seifert circles of $D$ as follows. A Seifert circle is a strongly negative circle, if it is adjacent to at least two negative crossings but adjacent to no positive crossings. A Seifert circle is a non-negative circle if it is not a strongly negative circle.

Let $L$ be an oriented link with $r$ components, and $D_{L}$ any diagram for $L$. Let $s_{\geq}\left(D_{L}\right)$ be the number of non-negative circles of $D_{L}$ and $s_{<}\left(D_{L}\right)$ be the number of strongly negative circles. In [16], Rudolph announced the stronger slice-Bennequin inequality,

$$
\chi_{s}(L) \leq\left(s_{\geq}\left(D_{L}\right)-s_{<}\left(D_{L}\right)\right)-w\left(D_{L}\right),
$$

and the author reproved it in [8]. By means of the above inequality and the inequality (1), the author showed the following inequality in [8]:

$$
u(L) \geq c_{s}(L) \geq \frac{1}{2}\left\{r-\left(s_{\geq}\left(D_{L}\right)-s_{<}\left(D_{L}\right)\right)+w\left(D_{L}\right)\right\} .
$$

Let $s(D)$ be the number of Seifert circles of a link diagram $D$. Let $L$ be an $r$-component link which has a closed braid diagram $D_{L}$. In [1], Bennequin conjectured the inequality,

$$
u(L) \geq \frac{1}{2}\left(r-s\left(D_{L}\right)+\left|w\left(D_{L}\right)\right|\right) .
$$

We shall call it the Bennequin unknotting inequality. In [15], by using a result of Kronheimer and Mrowka [9], [10], Rudolph showed the slice-Bennequin inequality,

$$
\chi_{s}(L) \leq s\left(D_{L}\right)-w\left(D_{L}\right) .
$$

It implies the Bennequin unknotting inequality by means of the inequality (1). In [18], Stoimenow showed that if the Bennequin unknotting inequality holds for any closed braid diagram, then it holds for any knot diagram. In fact, both of the Bennequin unknotting inequality and the slice-Bennequin inequality can be extended to any link diagram by the Vogel algorithm [20] or the Yamada algorithm [21]. The inequality (3) shows that an inequality stronger than that conjectured by Bennequin [1] actually holds.

A quasipositive link is an oriented link which has a closed quasipositive braid diagram, and a quasipositive braid is the product of conjugate braids with positive braids. In [15], Rudolph showed that the equality

$$
\chi_{s}(L)=s\left(D_{L}\right)-w\left(D_{L}\right)
$$

holds for any closed quasipositive braid diagram $D_{L}$ by a result of Kronheimer and Mrowka [9], [10]. A positive link is an oriented link which has a diagram with no negative crossings. In [12], [16], Nakamura and Rudolph showed that positive 
links are quasipositive, and that the above equality also holds for any positive link diagram. In [8] and [16], the stronger slice-Bennequin inequality is proved by using their result.

By means of the inequality (3), we determine not only the unknotting numbers but also the 4-dimensional clasp numbers of closed positive braids.

Theorem 2.1 ([8]). Let $L$ be an oriented link with $r$ components. We suppose that $L$ has a closed positive braid diagram $D_{L}$. Let $s\left(D_{L}\right)$ be the number of the Seifert circles and $x\left(D_{L}\right)$ be the number of the crossings of $D_{L}$. Then we have

$$
u(L)=c_{s}(L)=\frac{1}{2}\left(r-s\left(D_{L}\right)+x\left(D_{L}\right)\right) .
$$

The argument due to Rudolph in [14] gives a minimum unknotting operations for closed positive braid knots; if a positive braid representation has two consecutive letters equal, we change one of the crossings corresponding to them. This rule can be extended to closed positive braid links.

Remark 2.2. In [14], Rudolph defined the slice überschneidungszahl $\ddot{u}_{s}(K)$ of a knot $K$, and showed $\ddot{u}_{s}(K) \geq g^{*}(K)$, where $g^{*}(L)$ is the four-genus, the minimum genus for an oriented connected surface smoothly embedded in $D^{4}$ with boundary $L$. The slice überschneidungszahl and the four-dimensional clasp number are the same in the knot case. If $r=1$, that is, $L$ is an oriented knot, the equality $g^{*}(L)=\left(r-\chi_{s}(L)\right) / 2$ holds. We note that it may not hold if $r \geq 2$. In fact, in the case of the Hopf link, we have $g^{*}(L)=0$ and $\chi_{s}(L)=0$.

In [17], Shibuya defined the link invariant $c^{*}(L)$ as the minimum number of the double points for a transversely immersed surface in $D^{4}$ with genus 0 and boundary $L$. He called $c^{*}$ the clasp number and proved $g^{*} \leq c^{*} \leq u$ holds for any link. From definitions, we have $c^{*} \leq c_{s}$. If $L$ is a knot, the equality $c^{*}(L)=c_{s}(L)$ holds. But it does not hold in general, because if $L$ is the Hopf link, $c^{*}(L)=0$ and $c_{s}(L)=1$.

\section{The lowest degree of the Jones polynomial}

In order to prove Theorem 1.1, we review a result of Fiedler [4]. The n-string braid group $B_{n}$ is generated by $n-1$ standard generators $\sigma_{1}, \cdots, \sigma_{n-1}$ subject to the relations $\sigma_{i} \sigma_{i+1} \sigma_{i}=\sigma_{i+1} \sigma_{i} \sigma_{i+1}(1 \leq i \leq n-2), \sigma_{i} \sigma_{j}=\sigma_{j} \sigma_{i}$ if $|i-j|>1$. For $\beta \in B_{n}$, let $x_{i}^{+}(\beta)$ and $x_{i}^{-}(\beta)$ be the numbers of entries of $\sigma_{i}$ and $\sigma_{i}^{-1}$ in $\beta$ respectively. The writhe $w(\beta)$ is defined as $\sum_{i}\left(x_{i}^{+}(\beta)-x_{i}^{-}(\beta)\right)$. Let $x^{-}(\beta)$ be the number of negative crossings, that is $x^{-}(\beta)=\sum_{i} x_{i}^{-}(\beta)$. Let $\delta^{-}(\beta)$ be the number of different inverse generators $\sigma_{i}^{-1}$ which appear in the word $\beta$. Let $b^{-}(\beta)$ be the number of different inverse generators $\sigma_{i}^{-1}$ so that $x_{i}^{-}(\beta)>x_{i}^{+}(\beta)$. In [4], 
Fiedler proved the following theorem by results due to Kauffman [7].

Theorem 3.1 (Fiedler [4]). Let $\beta \in B_{n}$ be a braid and $L(\beta)$ be its corresponding oriented link. Then we have

$$
\min \operatorname{deg} V(L(\beta) ; t) \geq \frac{1}{2}(w(\beta)+1-n)-x^{-}(\beta)+\delta^{-}(\beta) .
$$

Moreover, we suppose that whenever an inverse generator $\sigma_{i}^{-1}$ appears in $\beta$ then there do not appear $\sigma_{i}, \sigma_{i-1}^{-1}, \sigma_{i+1}^{-1}$ in $\beta$. Then we have

$$
\min \operatorname{deg} V(L(\beta) ; t)=\frac{1}{2}(w(\beta)+1-n)-x^{-}(\beta)+b^{-}(\beta) .
$$

Proof of Theorem 1.1. Let $L$ be an oriented $r$-component link which has a closed positive braid diagram $D_{L}$. As Stoimenow commented in [18], the equality of Theorem 3.1 implies that the following equality holds for such a link $L$ :

$$
\min \operatorname{deg} V(L ; t)=\frac{1}{2}\left(x\left(D_{L}\right)+1-s\left(D_{L}\right)\right)
$$

Then by Theorem 2.1, the following equality holds for any closed positive braid link $L$ :

$$
\min \operatorname{deg} V(L ; t)=u(L)-\frac{1}{2}(r-1) .
$$

Furthermore combining it with the equality (4) and the inequality (1), we have $\min \operatorname{deg} V(L ; t)=c_{s}(L)-\frac{1}{2}(r-1)$ and min $\operatorname{deg} V(L ; t)=\frac{1}{2}\left(1-\chi_{s}(L)\right)$ for such a link $L$.

The Jones polynomial of a trivial link with $r$ components is $\left(-t^{1 / 2}-t^{-1 / 2}\right)^{r-1}$. But it is false that the minimum unknotting operation gives the lowest degree of the Jones polynomial for any closed positive braid link. The lowest degree term is given by the splicing operation corresponding to the crossing changing which we review on the paragraph after Theorem 2.1 .

Remark 3.2. In general, the equality $2 g^{*}=1-\chi_{s}$ does not hold.

Example 3.3. Let $T(p, q)$ be a $(p, q)$-torus link. We suppose that $p$ and $q$ are positive integers. Then the torus link $T(p, q)$ is a closed positive link. Let $d$ be the greatest common divisor of $p$ and $q$. In [8], the author has shown that the unknotting number of the $(p, q)$-torus link is $\frac{1}{2}\{(p-1)(q-1)+d-1\}$. Hence, by Theorem 1.1, we have

$$
\min \operatorname{deg} V(T(p, q) ; t)=\frac{1}{2}(p-1)(q-1)
$$


In fact, the Jones polynomial of the $(p, q)$-torus knot was obtained in [6] as follows;

$$
V(T(p, q) ; t)=\frac{t^{\frac{1}{2}(p-1)(q-1)}}{1-t^{2}}\left(1-t^{p+1}-t^{q+1}+t^{p+q}\right) .
$$

We note that $\frac{V(T(p, q) ; t)}{t^{(p-1)(q-1) / 2}}$ is a polynomial in $\mathbb{Z}[t]$ and does not vanish at $t=0$.

The following examples imply that Theorem 1.1 distinguishes some links from closed positive braids.

Example 3.4. It is well known that the knot which is denote by $6_{2}$ in [13] cannot be represented as a closed positive braid. We show this fact by Theorem 1.1. It is easy to show that the unknotting number of $6_{2}$ is 1 . By definition of the Jones polynomial or the table in [6], we have

$$
V\left(6_{2} ; t\right)=t^{-1}-1+2 t-2 t^{2}+2 t^{3}-2 t^{4}+t^{5} .
$$

Then the unknotting number of the knot $6_{2}$ is not equal to min $\operatorname{deg} V\left(6_{2} ; t\right)$. By Theorem 1.1, the knot $6_{2}$ cannot be represented as a closed positive braid.

Remark 3.5. The Conway polynomial $\nabla(L ; z) \in \mathbb{Z}[z]$ is an ambient isotopy invariant of oriented links $L$ and is defined by the following relations:

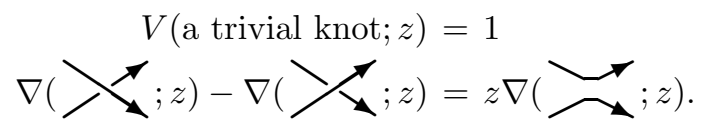

In [3], Cromwell showed that any positive link has a positive Conway polynomial. In [19], Van Buskirk showed that, if $K$ is a closed positive braid knot, then

$$
\nabla(K ; z)=z^{2 m}+a_{2 m-2} z^{2 m-2}+\cdots+a_{4} z^{4}+a_{2} z^{2}+1,
$$

where $\left(\begin{array}{c}m \\ k\end{array}\right) \leq a_{2(m-k)} \leq\left(\begin{array}{c}2 m-k \\ k\end{array}\right)$. These facts imply that the knot $6{ }_{2}$ cannot be represented as a closed positive braid, since we have $\nabla\left(6_{2} ; z\right)=1-z^{2}-z^{4}$.

Example 3.6. Let $8_{20}$ ! be the mirror image of the knot denoted by $8_{20}$ in [13]. The knot $8_{20}$ ! is the closure of the quasipositive braid $\sigma_{1}^{3} \sigma_{2} \sigma_{1}^{-3} \sigma_{2} \in B_{3}$ as listed in the table in [6]. The Conway polynomial of $8_{20}$ ! is $\nabla\left(8_{20} ! ; z\right)=1+2 z^{2}+z^{4}$. By definition of the Jones polynomial or the table in [6], we have

$$
V\left(8_{20} ! ; t\right)=-t^{5}+t^{4}-t^{3}+2 t^{2}-t+2-t^{-1}
$$

Hence the knot $8_{20}$ ! cannot be represented as a closed positive braid. In fact we can show this fact by Theorem 2.1 , since the knot $8_{20}$ ! is a non-trivial slice knot.

Remark 3.7. Let $5_{2}$ ! be the mirror image of the knot denoted by $5_{2}$ in [13]. The knot $5_{2}$ ! is positive. The Conway polynomial of $5_{2}$ ! is $\nabla\left(5_{2} ! ; z\right)=1+2 z^{2}$. Hence 
the knot $5_{2}$ ! cannot be represented as a closed positive braid, though the Jones polynomial of $5_{2}$ ! is $V\left(5_{2} ! ; z\right)=-t^{6}+t^{5}-t^{4}+2 t^{3}+t$ and $\min \operatorname{deg} V\left(5_{2} ! ; z\right)=1=$ $u\left(5_{2} !\right)$.

\section{Relations for a quasipositive link}

Let $\beta \in B_{n}$ be a braid and $L(\beta)$ be its corresponding oriented link. We suppose that whenever an inverse generator $\sigma_{i}^{-1}$ appears in $\beta$ then there do not appear $\sigma_{i}, \sigma_{i-1}^{-1}, \sigma_{i+1}^{-1}$ in $\beta$. By the equality of Theorem 3.1 and the slice-Bennequin inequality, we have

$$
\min \operatorname{deg} V(L(\beta) ; t) \leq \frac{1}{2}\left(1-\chi_{s}(L)\right)-x^{-}(\beta)+b^{-}(\beta) .
$$

By means of the stronger slice-Bennequin inequality and Theorem 3.1, if $\sigma_{1}^{-1}$ appears in $\beta$ at most once and $\sigma_{n-1}^{-1}$ appears in $\beta$ at least twice, then we have

$$
\min \operatorname{deg} V(L(\beta) ; t) \leq \frac{1}{2}\left(-1-\chi_{s}(L)\right)-x^{-}(\beta)+b^{-}(\beta)
$$

and if both of $\sigma_{1}^{-1}$ and $\sigma_{n-1}^{-1}$ appear in $\beta$ at least twice, then we have

$$
\min \operatorname{deg} V(L(\beta) ; t) \leq \frac{1}{2}\left(-3-\chi_{s}(L)\right)-x^{-}(\beta)+b^{-}(\beta) .
$$

In [4], Fiedler gave some conjectures. We consider one of them. Let $\beta \in B_{n}$ be a braid and $L(\beta)$ be its corresponding oriented link. If $\beta$ is of the form $\beta=$ $w_{1} \sigma_{i_{1}}^{ \pm 1} w_{1}^{-1} \cdots w_{k} \sigma_{i_{k}}^{ \pm 1} w_{k}^{-1}, \beta$ is given in a band representation. A factor of the form $w_{j} \sigma_{i_{j}} w_{j}^{-1}$ is called positive band and a factor of the form $w_{j} \sigma_{i_{j}}^{-1} w_{j}^{-1}$ is called negative band.

Conjecture 4.1 (Fiedler [4]). Let $\beta \in B_{n}$ be a braid given in a band representation. Let $d^{-}(\beta)$ be the number of negative bands of a band representation $\beta$. Then the following inequality holds:

$$
\min \operatorname{deg} V(L(\beta) ; t) \leq \frac{1}{2}(w(\beta)+1-n)+d^{-}(\beta) .
$$

By the slice-Bennequin inequality and the inequality (1), if the above conjecture would be true, the following inequality would hold for any quasipositive link $L$ with $r$ components:

$$
\min \operatorname{deg} V(L(\beta) ; t) \leq \frac{1}{2}\left(1-\chi_{s}(L)\right) \leq u(L)-\frac{1}{2}(r-1)
$$

Remark 4.2. Example 3.6 implies the equality min $\operatorname{deg} V(L(\beta) ; t)=\frac{1}{2}\left(1-\chi_{s}(L)\right)$ does not hold for general quasipositive links. 


\section{References}

[1] D. Bennequin, Entrelacement et équations de Pfaff, Astérisque 107-108 (1983), 87-161.

[2] M. Boileau and C. Weber, Le probléme de J. Milnor sur le nombre gordien des nœuds algébriques, L'Enseign. Math. 30 (1984), 173-222.

[3] P. Cromwell, Homogeneous links, J. London Math. Soc. 39 (1989), 535-552.

[4] T. Fiedler, On the degree of the Jones polynomial, Topology 30 (1991), 1-8.

[5] V. Jones, A polynomial invariant for knots via von Neumann algebras, Bull. Amer.Math. Soc. 12 (1985), 103-111.

[6] V. Jones, Hecke algebra representations of braids groups and link polynomials, Ann. of Math. 126 (1987), 335-388.

[7] L. Kauffman, State models and the Jones polynomial, Topology 26 (1987), 395-407.

[8] T. Kawamura, On unknotting numbers and four-dimensional clasp numbers of links, to appear in Proc. Amer. Math. Soc. 130 (2002), 243-252.

[9] P. Kronheimer and T. Mrowka, Gauge theory for embedded surfaces, I, Topology 32 (1993), 773-826.

[10] P. Kronheimer and T. Mrowka, Gauge theory for embedded surfaces, II, Topology 34 (1995), 37-97.

[11] J. Milnor, Singular points of complex hypersurfaces, Ann. of Math. Studies 61 (1968), Princeton Univ. Press.

[12] T. Nakamura, Four-genus and unknotting number of positive knots and links, Osaka J. Math 37 (2000), 441-452.

[13] D. Rolfsen, Knots and links, Mathematics Lecture Series 7, Publish or Perish, Inc., Berkeley, Calif., 1976.

[14] L. Rudolph, Braided surfaces and Seifert ribbons for closed braids, Comment. Math. Helvetici $\mathbf{5 8}$ (1983), 1-37.

[15] L. Rudolph, Quasipositivity as an obstruction to sliceness, Bull. Amer. Math. Soc. 29 (1993), 51-59

[16] L. Rudolph, Positive links are strongly quasipositive, Geometry and Topology Monographs 2 (1999): Proc. of the Kirbyfest, 555-562.

[17] T. Shibuya, Some relations among various numerical invariants for links, Osaka J. Math. 11 (1974), 313-322.

[18] A. Stoimenow, Positive knots, closed braids and the Jones polynomial, preprint, 1999.

[19] J. Van Buskirk, Positive knots have positive Conway polynomials, Knot theory and manifolds, 146-159, Lect. Notes Math. 1144, Springer, Berlin, 1985.

[20] P. Vogel, Representation of links by braids: A new algorithm, Comment. Math. Helv. 65 (1990), 104-113.

[21] S. Yamada, The minimal number of Seifert circles equals the braid index of a link, Invent. Math. 89 (1987), 347-356.

Tomomi Kawamura

Graduate School of Mathematical Sciences

University of Tokyo

3-8-1 Komaba Meguro-ku

Tokyo 153-8914

Japan

e-mail: kawamura@ms.u-tokyo.ac.jp
Current address:

Department of Mathematics

Aoyama Gakuin University

6-16-1, Chitosedai, Setagaya

Tokyo 157-8572

Japan

e-mail: tomomi@gem.aoyama.ac.jp

(Received: September 19, 2000) 